\title{
Synthesis of monodispersed palladium nanoparticles to study structure sensitivity of solvent-free selective hydrogenation of 2-methyl-3-butyn-2-ol
}

\author{
Natalia Semagina ${ }^{\mathrm{a}}$, Albert Renken ${ }^{\mathrm{a}}$, Daniele Laub ${ }^{\mathrm{b}}$, Lioubov Kiwi-Minsker ${ }^{\mathrm{a}, *}$ \\ a Ecole Polytechnique Federale de Lausanne, GGRC-ISIC-EPFL, Station 6, CH-1015 Lausanne, Switzerland \\ ${ }^{\mathrm{b}}$ CIME-SB-EPFL, Lausanne, Switzerland
}

Received 10 October 2006; revised 7 December 2006; accepted 8 December 2006

Available online 22 January 2007

\begin{abstract}
A novel method for isolation of monodispersed Pd nanoparticles from a reverse microemulsion was developed using hydrocarbon evaporation and methanol-assisted particle purification from a surfactant. Fcc Pd nanoparticles of 6, 8, 11, and 13 nm in diameter were isolated from water/ AOT/isooctane mixture and used to study a size effect during solvent-free hydrogenation of 2-methyl-3-butyn-2-ol to 2-methyl-3-buten-2-ol. The initial TOF calculated per mole of surface palladium atoms was duplicated when particle size was increased from 6 to 13 nm but remained constant when accounted per number of specific Pd atoms on Pd(111) facets. Selectivity to olefinic alcohol was not size-dependent, but an increase in particle size decreased the byproduct ratio of dimers to saturated alcohol. Acetylenic alcohol hydrogenation is shown to be a structure-sensitive but size-independent reaction for Pd particles with size of 6-13 $\mathrm{nm}$. The work shows also that the Pd size controlled the reaction rate and the byproduct distribution.
\end{abstract}

(c) 2007 Elsevier Inc. All rights reserved.

Keywords: Palladium nanoparticle; Hydrogenation; 2-Methyl-3-butyn-2-ol; Microemulsion; Size effect; Structure sensitivity

\section{Introduction}

Structure sensitivity is of great interest in heterogeneous catalysis. Metal particle morphology and size influence the turnover frequency (TOF) and selectivity due to well-known electronic and geometric effects [1-6]. The relative ratio of surface atom types (vertex, edge, and facet atoms) changes substantially with varying particle size; large particles have mainly large crystal planes with atoms of high coordination number, whereas metal atoms with low coordination numbers constitute small particles [6,7]. The reactions that require a special type of surface atom or an ensemble of surface atoms are strongly affected by the variation in the particle size, for example, Pdcatalyzed hydrogenations or Suzuki reactions $[8,9]$.

The main requirement in studying the structure sensitivity of catalytic reactions is that the particle size should be varied in the absence of other influences [7]. The use of different metal pre-

\footnotetext{
* Corresponding author. Fax: +41216936190.

E-mail address: lioubov.kiwi-minsker@epfl.ch (L. Kiwi-Minsker).
}

cursors, catalyst supports, or preparation conditions had led to catalysts that are intrinsically different from each other besides in particle size $[2,7,10]$. Colloid preparation techniques may provide suitable catalysts for such studies [2]. Among these, the reverse microemulsion (ME) technique is of special interest. Reversed (water-in-oil) MEs are thermodynamically stable, optically isotropic dispersions of water and oil consisting of nanosized domains of water in oil, stabilized by an interfacial film of surface active molecules [11]. The water droplets can be considered spatially confined nanoreactors of 5-100 nm for the formation of monodispersed particles with a standard deviation less than $\pm 10 \%$. In this case, monodispersed nanoparticles with controlled sizes are synthesized [12-17]. Through this approach, catalytic metal nanoparticles of different sizes are prepared in the same manner by simply varying the waterto-surfactant ratio in MEs.

ME-derived nanoparticles are widely used in catalytic reactions, mainly as a nanoparticle-containing MEs per se or deposited onto supports. The use of ME itself [18-25] implies the addition of extra components to the catalytic reaction mixture (hydrocarbon, water, surfactant, excess of a metal re- 
ducing agent). This leads to an increase of the reaction volume, and a catalytic reaction may be affected through "medium" and "solubilization" effects [26,27]. Multicomposition of ME also does not allow conducting solvent-free reactions. Several attempts have been made to purify nanoparticles from the ME components. Hydrocarbon evaporation [11,28] or freezedrying/sublimation [29] did not allow elimination of surfactant molecules, whereas cross-flow ultrafiltration [30] resulted in concentrated nanoparticle suspension. Nanoparticles also may be deposited onto solid support by mixing the ME with a support. This step is usually followed by calcination $[12,16,17,21$, 31-41]. However, these heterogeneous catalysts can be hardly applied for size effect studies because of specific particlesupport interactions [21] and the sintering of the particles at high temperatures during the calcination step.

Here we propose a simple method of preparing monodispersed metal nanoparticles for size effect studies. It implies purification of nanoparticles synthesized in reverse ME and their dispersion in a pure reactant. The method allows us to obtain monodispersed nanoparticles with controlled sizes and to recover solvent/surfactant from MEs.

Hydrogenation of 2-methyl-3-butyn-2-ol (MBY) to 2methyl-3-buten-2-ol (MBE) was taken as a model reaction because it is important in the synthesis of vitamins and perfumes. The reaction yields a number of byproducts (see Fig. 1) and thus allows the study of the influence of $\mathrm{Pd}$ particle size on both activity and selectivity.

Data on size effects in alkyne hydrogenation are rather controversial, although most studies have shown that an increase in metal dispersion decreases TOF [6]. The size effect in alkyne Pd-catalyzed hydrogenation was first reported by Boitiaux et al. [42]. Lower selectivity and more than a 10-fold decrease in TOF were observed when the metal dispersion increased from 20 to $100 \%$. The low activity of small metal particles (with a dispersion of $>20 \%$ ) is usually explained by the strong complexation of the highly unsaturated electron-rich alkyne to the electron-deficient atoms with low coordination numbers on small particles. Moreover, the $\beta$ - $\mathrm{PdH}$ phase disappears with decreasing metal particle size [43]. Some studies have shown this phase to be responsible for the direct alkyne hydrogenation to alkane [5], whereas others did not find this detrimental effect $[6,44]$. Pd-catalyzed hydrogenation of acetylenic alcohols

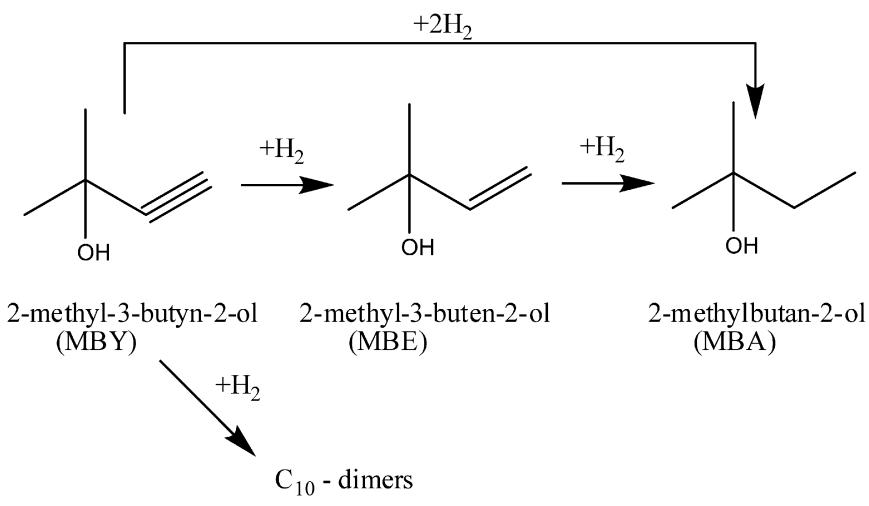

Fig. 1. Reaction pathways for the conversion of 2-methyl-3-butyn-2-ol. is also known to be a structure-sensitive reaction. However, the size dependence is strongly affected by the catalyst preparation. When Pd nanoparticles were stabilized by poly(vinylpyrrolidone), an increase in the particle size from 5 to $7 \mathrm{~nm}$ led to a twofold decrease in TOF and an $8 \%$ increase in selectivity during the hydrogenation of 2-butyne-1,4-diol [10]. Pd nanoparticles deposited on activated carbon fiber fabrics exhibited lower TOFs and selectivities at higher dispersions $[45,46]$. Pd nanoparticles $7.5 \mathrm{~nm}$ in size showed sixfold higher activity and $14 \%$ higher selectivity in MBY hydrogenation compared with 2.5-nm particles [47,48], but the reactions were carried out with the supported particles, and no tests for the kinetic regime were reported.

No studies on the size effect in hydrogenation of acetylenic alcohols have been reported for particles larger than $7.5 \mathrm{~nm}$. Usually, the structure-sensitive reactions are considered to not reveal size dependence above $5 \mathrm{~nm}$ owing to the relatively small variation of the portion of the specific atoms (edge and face) on the nanoparticle surface [7]. Thus, the aim of this work was to elaborate a method for monodispersed Pd nanoparticle preparation and to study size effect in 2-methyl-3-butyn-2-ol hydrogenation using the nonsupported Pd nanoparticles 6-13 $\mathrm{nm}$ in size.

\section{Experimental}

\subsection{Materials}

Tetraamminepalladium(II) chloride monohydrate (99.99+\%; Aldrich), isooctane (2,2,4-trimethylpentane, extra dry, with molecular sieve, water $<30$ ppm; Acros Organics), fumic hydrochloric acid (purum, p.a.; Fluka), nitric acid (puriss., p.a.; Fluka), hydrazinium hydroxide (for synthesis; Merck), 32\% ammonium solution (extra pure; Merck), methanol (for analysis; ReactoLab C.A.), 2-methyl-3-butyn-2-ol (purum, $\geqslant 99 \%$; Fluka), and hydrogen ( $\geqslant 99.99 \%$ purity; Carbagas, Switzerland) were used as received. AOT (sodium di-2-ethylhexylsulfosuccinate, or docusate sodium salt, purum, $\geqslant 96 \%$; Fluka) was vacuum-dried for $24 \mathrm{~h}$ at $333 \mathrm{~K}$ directly before use. All glassware was air-dried at $393 \mathrm{~K}$. Demineralized bidistilled water was used throughout this work. Pd black (Aldrich) was reduced at $573 \mathrm{~K}$ for $3 \mathrm{~h}$ immediately before use.

\subsection{Pd nanoparticles: preparation and characterization}

A general scheme for preparation of metal nanoparticles for size-effect studies is presented in Fig. 2. Pd nanoparticles were synthesized in reverse ME of water/AOT/isooctane at different water-to-surfactant ratios $(3,4,5$, and 7$)$ as described previously [49]. The aqueous solution of $\mathrm{PdCl}_{2}\left(\mathrm{NH}_{3}\right)_{4}(0.05 \mathrm{M})$ was used as a metal precursor ( $\mathrm{pH} 9$, adjusted with ammonia), and the solution of hydrazine hydrate $(3 \mathrm{M})$ as a reducing agent, giving a hydrazine-to-Pd molar ratio of 60 to ensure precursor reduction. The reverse ME containing metal precursor or reducing agent was prepared by injecting the required amounts of the corresponding aqueous solution into an isooctane solution of 


\section{Pd-nanoparticles}

\section{in reverse microemulsion}

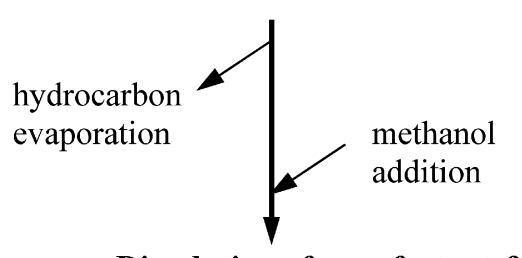

Dissolution of a surfactant $\&$

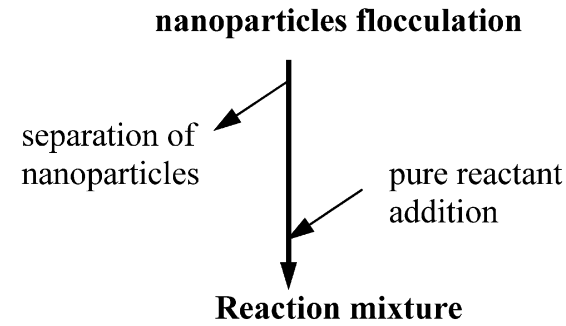

with Pd nanoparticles

Fig. 2. Scheme of nanoparticles preparation for catalytic application.

AOT $(0.35 \mathrm{M})$ and then mixing for a few minutes for the preparation of Pd nanoparticles. As an example, for the preparation of nanoparticle-containing MEs with a water-to-surfactant ratio of 7, $15.45 \mathrm{~g}$ AOT, total isooctane volume of $0.1 \mathrm{~L}, 2.2 \mathrm{~mL}$ of aqueous Pd precursor, and $2.2 \mathrm{~mL}$ of reductant were used.

After $1 \mathrm{~h}$ of reduction at room temperature, isooctane was evaporated in a rotary evaporator at $323 \mathrm{~K}$, and the nanoparticles were purified from an excess of surfactant by addition of methanol followed by centrifugation $(3 \times 30 \mathrm{~mL})$. Precipitated Pd nanoparticles after centrifugation were immediately redispersed under ultrasound in the substrate (MBY) for the hydrogenation reaction and used in catalytic runs. Pd colloids remained stable for at least 1 month without any special precautions.

The Pd content in the colloidal solution was determined after it was mixed with hot nitric acid by atomic absorption spectroscopy (AAS) at $247.6 \mathrm{~nm}$ via a Shimadzu AA-6650 spectrometer with an air-acetylene flame. Pd particle sizes in MBY were determined by high-resolution transmission electron microscopy (HRTEM) using a Philips CM-300 FEG instrument at $300 \mathrm{kV}$. The sample for TEM analysis was prepared by placing a drop of the suspension onto the carbon film on the copper grid and evaporating it in air at room temperature. For each sample, more than 150 particles from different parts of the grid were used to determine the particle size and particle size distribution.

XRD analysis was carried out in a Siemens D 500 diffractometer using $\mathrm{Cu} K \alpha$ radiation. The spectra were recorded at $10 \mathrm{~s} / \mathrm{step}, 2 \theta$ step size of $0.02^{\circ}$ in a $2 \theta$ range of $35^{\circ}-70^{\circ}$. The sample was prepared by the incipient wetness impregnation of $\mathrm{Al}_{2} \mathrm{O}_{3}$ with Pd colloids in 2-methyl-3-butyn-2-ol synthesized at a water-to-surfactant ratio of 7 and dried in vacuo at $333 \mathrm{~K}$ overnight.

To determine a degree of purification of Pd nanoparticles from the ME constituents, isolated nanoparticles were dried in vacuo at $333 \mathrm{~K}$ overnight. Pd content in the obtained pow- der was determined by AAS after dissolution in nitric acid. To examine a surface composition, energy-dispersive X-ray analysis (EDX) of the isolated nanoparticles was performed using a Philips CM-300 FEG instrument at $300 \mathrm{kV}$. The quantitative surface composition was controlled by XPS using an Axis Ultra ESCA system (Kratos, Manchester) with monochromated Al$K \alpha$ radiation $(1486.6 \mathrm{eV})$ and an $\mathrm{X}$-ray power of $150 \mathrm{~W}$. The binding energy scale was referenced against a $\mathrm{C} 1 \mathrm{~s}=285.0 \mathrm{eV}$ line.

Metal dispersion of Pd black was measured by pulse adsorption of $\mathrm{CO}(3 \% \mathrm{CO}$ in $\mathrm{He}$ ) performed at $323 \mathrm{~K}$ in a Micromeritics AutoChem 2910. The sample was prereduced at $573 \mathrm{~K}$ in a flow of $\mathrm{H}_{2}$. A stoichiometry of $\mathrm{CO} / \mathrm{Pd}=0.6$ and a $\mathrm{Pd}$ surface density of $1.2 \times 10^{19}$ atoms $/ \mathrm{m}^{2}$ was used for calculations.

\subsection{Hydrogenation experiments}

Hydrogenations were carried out in a batch stainless steel reactor $(250 \mathrm{~mL}$ autoclave, Buchi AG, Uster, Switzerland) equipped with a heating jacket, hydrogen supply system, and 8-blade disk turbine impeller. At the working temperature, the reactor was filled with the reaction mixture and the catalyst, flushed with $\mathrm{N}_{2}$, and stirred for $10 \mathrm{~min}$ to equalize the temperature. Then the reactor was flushed with hydrogen and pressurized. The pressure in the reactor was kept constant during the course of the reaction.

The experiments were carried out at $333 \mathrm{~K}$ and $0.28 \mathrm{MPa}$ pressure under intensive stirring at $2000 \mathrm{rpm}$. A 0.2-L (172.2 g) sample of 2-methyl-3-butyn-2-ol and a 1.44-mg sample of Pd were used, for a substrate-to-Pd molar ratio of 150,000. For Pd black, $30 \mathrm{mg}$ of catalyst was used to give a reasonable reaction rate.

The samples were periodically withdrawn from the reactor and analyzed by gas chromatography (GC) using a Perkin Elmer Auto System XL equipped with a 30-m Stabilwax (Crossbond Carbowax-PEG, Restek) $0.32-\mathrm{mm}$ capillary column with a $0.25-\mu \mathrm{m}$ coating. The carrier gas $(\mathrm{He})$ pressure was $0.1 \mathrm{MPa}$. Injector and flame ionization detector temperatures were $473 \mathrm{~K}$ and $523 \mathrm{~K}$, respectively. The oven temperature was maintained at $323 \mathrm{~K}$ for $4 \mathrm{~min}$, then increased to $473 \mathrm{~K}$ at a ramp rate of $30 \mathrm{~K} / \mathrm{min}$. The $\mathrm{GC}$ analysis conditions also allowed detection of the dimerized byproducts formed during the reaction. The yield of the reaction mixture components $Y_{i}$ (where $i$ represents MBY, MBE, MBA, or dimers) was calculated from the peak areas assuming similar GC response factors. Selectivities to MBE, MBA, and dimers were defined as $S_{i}=Y_{i} \times X^{-1} \times 100 \%$, where $X$ is a MBY conversion. Values for the initial selectivity were obtained by extrapolation of selectivity-conversion dependence. As a measure of activity, initial TOF was used as $\mathrm{mol}_{\mathrm{MBY}} \mathrm{mol}_{\text {Pd surface atoms }}^{-1} \mathrm{~s}^{-1}$ (if not otherwise specified). Amount of Pd surface atoms was calculated using the statistics for face-centered cubic (fcc) nanoparticles of known size [7]. The values of initial activity and selectivity were used because this is a common approach in size-effect studies [8]. These values represent catalytic properties without the influence of catalyst deactivation. 


\section{Results and discussion}

\subsection{Characterization of Pd nanoparticles}

The parameters of the synthesized Pd nanoparticles are summarized in Table 1. The nanoparticle size increased with water-to-surfactant ratio, and the values were in agreement with the values reported for $\mathrm{Pd}$ particles in water/AOT/isooctane ME [49]. This indicates that no coagulation took place during particle purification. Synthesized particles were also characterized by a narrow size distribution with a deviation of $<25 \%$. Fig. 3 shows the TEM images and histograms of the particle size distribution for Pd nanoparticles synthesized at water-tosurfactant ratios of 3 and 7.

The crystalline phase of the synthesized particles was confirmed by HRTEM (see, e.g., Fig. 4 for the Pd11 sample) and $\mathrm{XRD}$, which revealed the presence of pure palladium with characteristic peaks at $2 \theta$ of $40^{\circ}, 46^{\circ}$, and $68^{\circ}$, corresponding to $\{111\},\{200\}$, and $\{220\}$ planes of an fcc lattice. An XPS spectrum of the $\mathrm{Pd} 3 \mathrm{~d}$ region $\left(\mathrm{Pd} 3 \mathrm{~d}_{5 / 2}\right.$ and $\left.\mathrm{Pd} 3 \mathrm{~d}_{3 / 2}\right)$ for the $\mathrm{Pd} 13$

Table 1

Main parameters of the synthesized Pd nanoparticles

\begin{tabular}{llcl}
\hline $\begin{array}{l}\text { Catalyst } \\
\text { designation }\end{array}$ & $\begin{array}{l}\text { Water-to-surfactant } \\
\text { ratio }(\mathrm{mol} / \mathrm{mol})\end{array}$ & $\begin{array}{l}\text { Particle size and } \\
\text { standard deviation }^{\mathrm{a}}(\mathrm{nm})\end{array}$ & $\begin{array}{l}\text { Dispersion }^{\mathrm{b}} \\
(\%)\end{array}$ \\
\hline Pd6 & 3 & $6.1 \pm 1.0$ & 26.1 \\
Pd8 & 4 & $8.2 \pm 2.0$ & 20.3 \\
Pd11 & 5 & $10.8 \pm 1.4$ & 15.2 \\
Pd13 & 7 & $12.7 \pm 1.4$ & 13.1 \\
\hline
\end{tabular}

a Determined by HRTEM.

b Using the statistics for fcc cubo-octahedron max-B 5 crystals [7]. sample is shown in Fig. 5, along with the deconvolution results. Metallic $\mathrm{Pd}$ represents $70 \%$ of total surface $\mathrm{Pd}$, with the remaining $30 \%$ attributed to the ionic species originated from $\mathrm{PdO}$ and $\mathrm{PdO}_{2}$.

Quantitative surface composition of the isolated nanoparticles, Pd13, determined by XPS is represented by $\mathrm{O}(40 \mathrm{wt} \%)$, $\mathrm{Na}(23 \mathrm{wt} \%), \mathrm{C}(19 \mathrm{wt} \%), \mathrm{S}(15 \mathrm{wt} \%)$, and Pd (3 wt \%). Four first elements are attributed to the presence of the surfactant at an S:O:Na weight ratio of 1:3:1, which is nearly the same as that determined in the sample by XPS. EDX analysis also confirmed the presence of AOT and absence of nitrogen and chlorine, indicating that the Pd colloids were free of the metal precursor and the reducing agent. The Pd content in the dried Pd nanoparticles powder was determined by AAS to be $15 \mathrm{wt} \%$, which is the same as found by XPS taking into account a Pd dispersion of

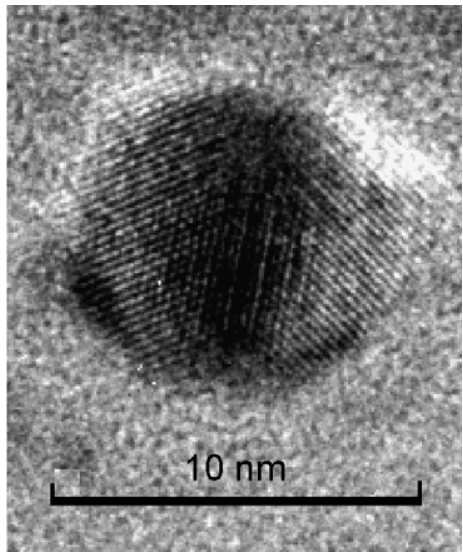

Fig. 4. HRTEM image of a nanoparticle from Pd11 catalyst.
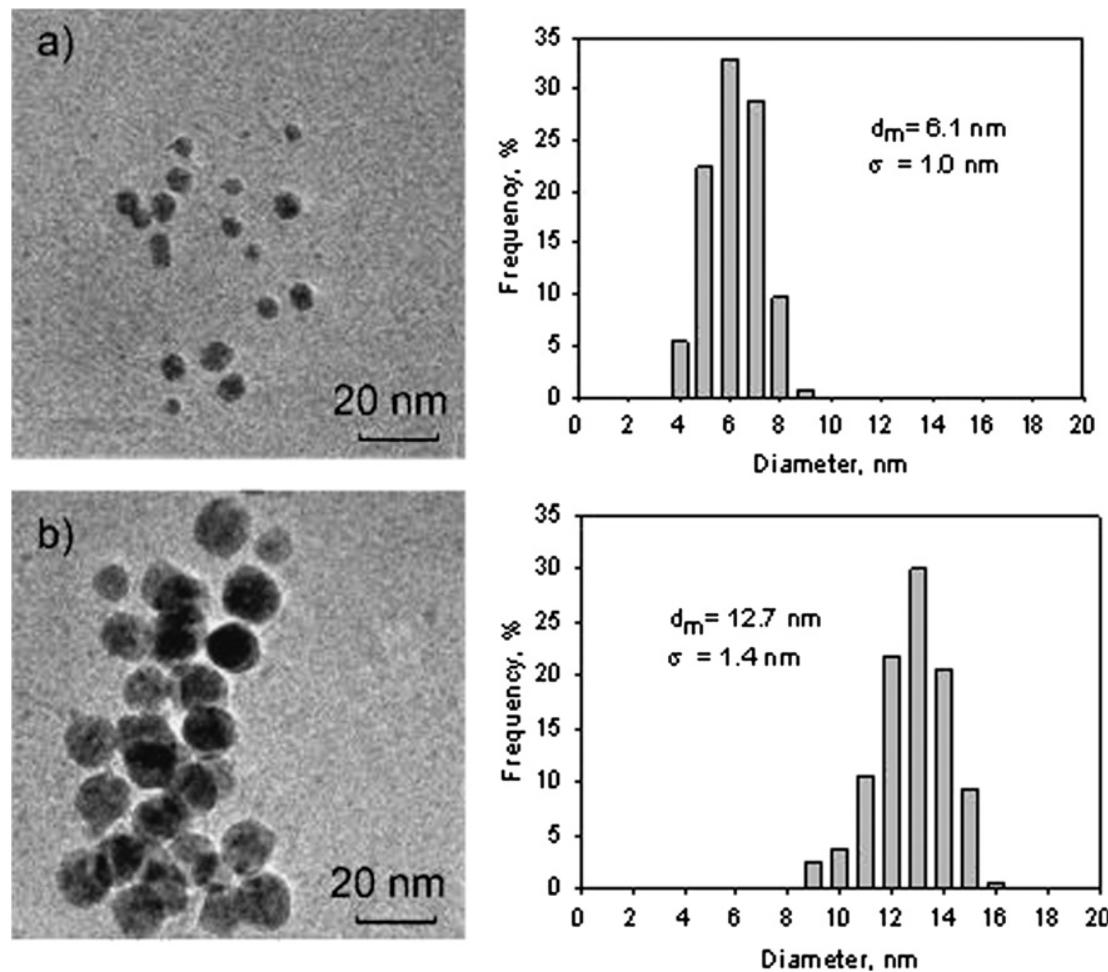

Fig. 3. TEM images and histograms of the particle size distribution for Pd6 (a) and Pd13 (b) catalysts. 


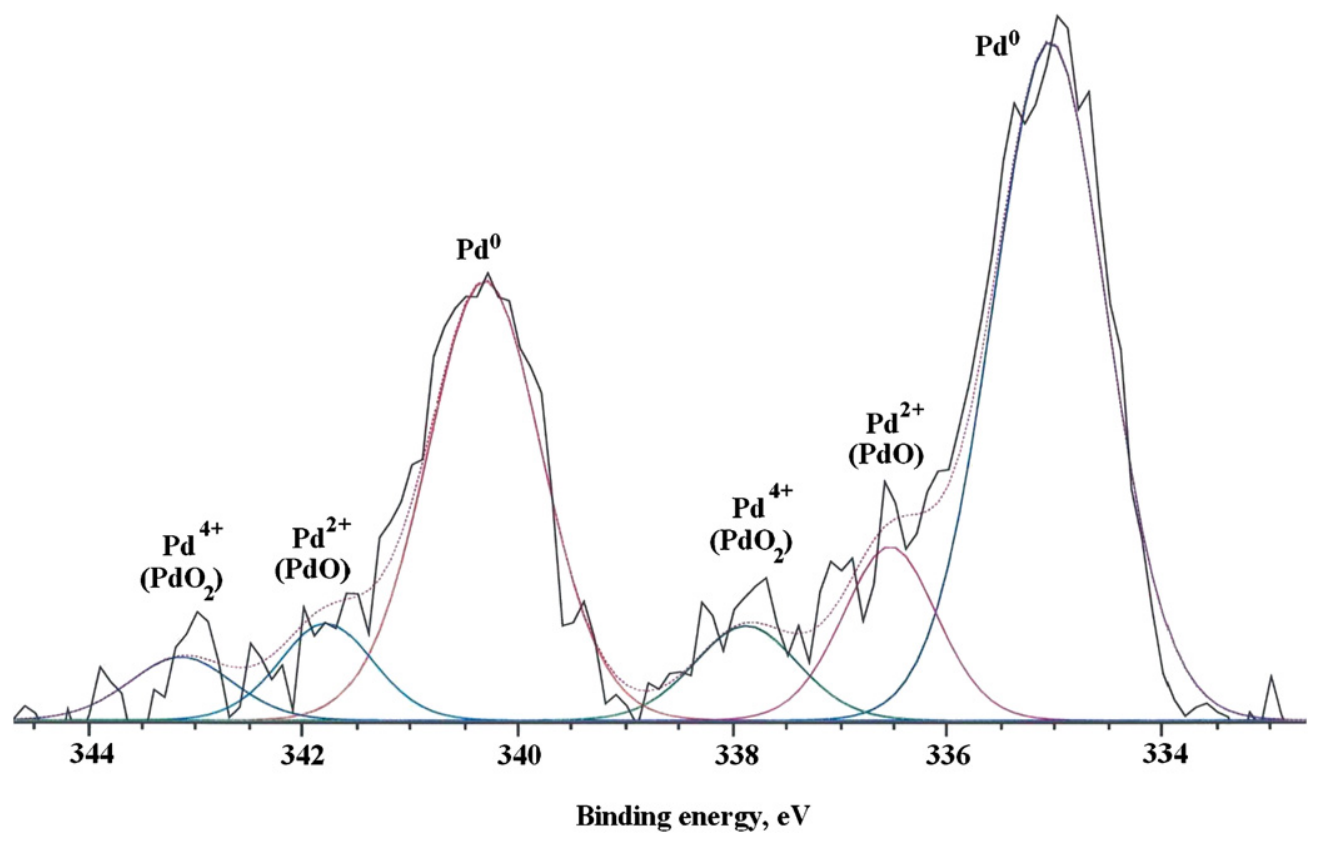

Fig. 5. XPS of the Pd 3d region of the isolated Pd nanoparticles (Pd13).

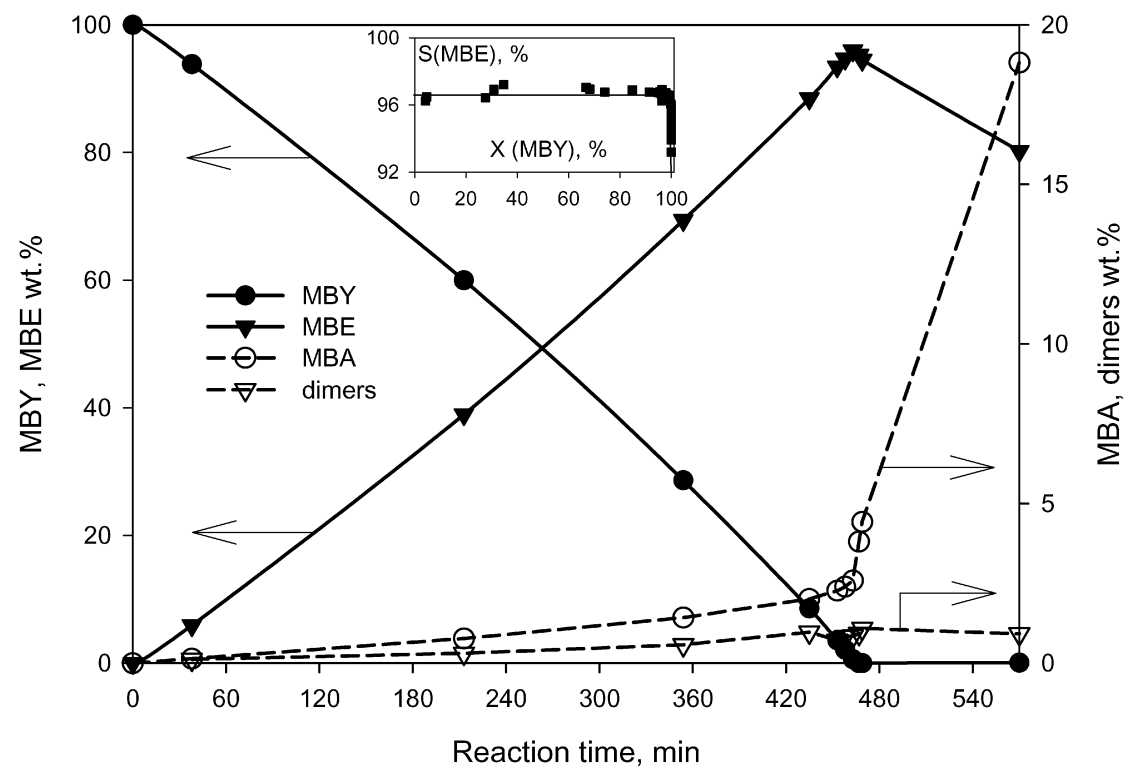

Fig. 6. Kinetic curves of MBY hydrogenation with Pd13 catalyst. Inset: MBE selectivity vs MBY conversion. Reaction conditions: 172.2 g MBY, 1.44 mg Pd, $333 \mathrm{~K}, 0.28 \mathrm{MPa}, 2000 \mathrm{rpm}$.

$13.1 \%$ (Table 1). Another $75 \mathrm{wt} \%$ is attributed to the surfactant residuals; AOT weight content in the powder may be 235 times lower than in the initial ME. This again correlates with XPS and EDX results showing a $~ 200$-fold lower weight amount of sulfur and oxygen than initially found. All of the findings confirm that $>99.5 \mathrm{wt} \%$ of the surfactant were removed by washing with methanol.

\subsection{Catalytic hydrogenations: size effect}

Typical kinetic curves of MBY hydrogenation with monodispersed $\mathrm{Pd}$ nanoparticles are presented in Fig. 6, along with MBE selectivity versus MBY conversion. The negative ap- parent order with respect to MBY was in agreement with the reported data for Pd-catalyzed hydrogenation of acetylenic alcohols [50]. MBA appears at the very beginning of the reaction, confirming the presence of a parallel path of the direct MBY hydrogenation to MBA. When MBY is consumed, the rate of dimer formation drops to zero. The same features were observed for MBY hydrogenation over the $\mathrm{Pd}-\mathrm{Pb} / \mathrm{CaCO} 3$ catalyst.

The dependence of the MBY hydrogenation rate on particle size shows that the initial TOF calculated per surface Pd atom grows with particle size. It increased twice when the diameter changed from 6 to $13 \mathrm{~nm}$ (Fig. 7). This indicates that Pd atoms on the surface of a larger particle are more active than those on a smaller particle, assuming that all particles take part in the 


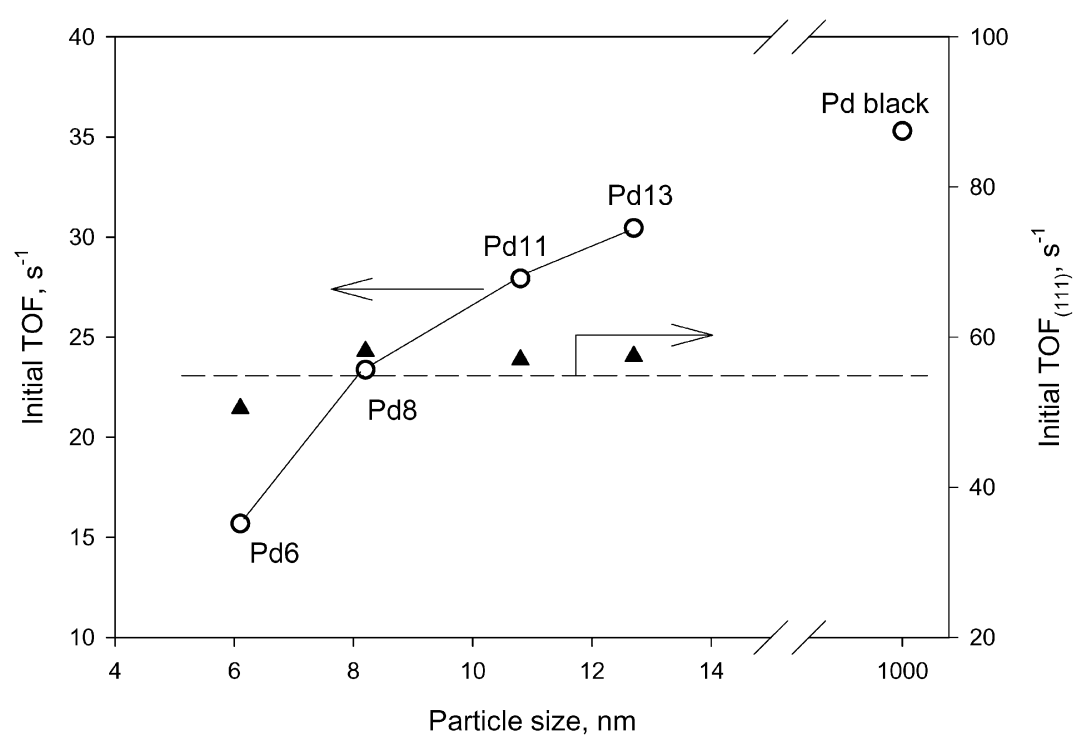

Fig. 7. Size dependence of TOF calculated per total surface atoms number $\left(\mathrm{O}\right.$, left axis) and per the number of $\mathrm{C}_{9}^{3}$ atoms of fcc cubo-octahedron max- $\mathrm{B}_{5}$ crystal [7] ( $\Lambda$, right axis) in MBY hydrogenation. Reaction conditions: see Fig. 6, for Pd black $30 \mathrm{mg}$ of catalyst.

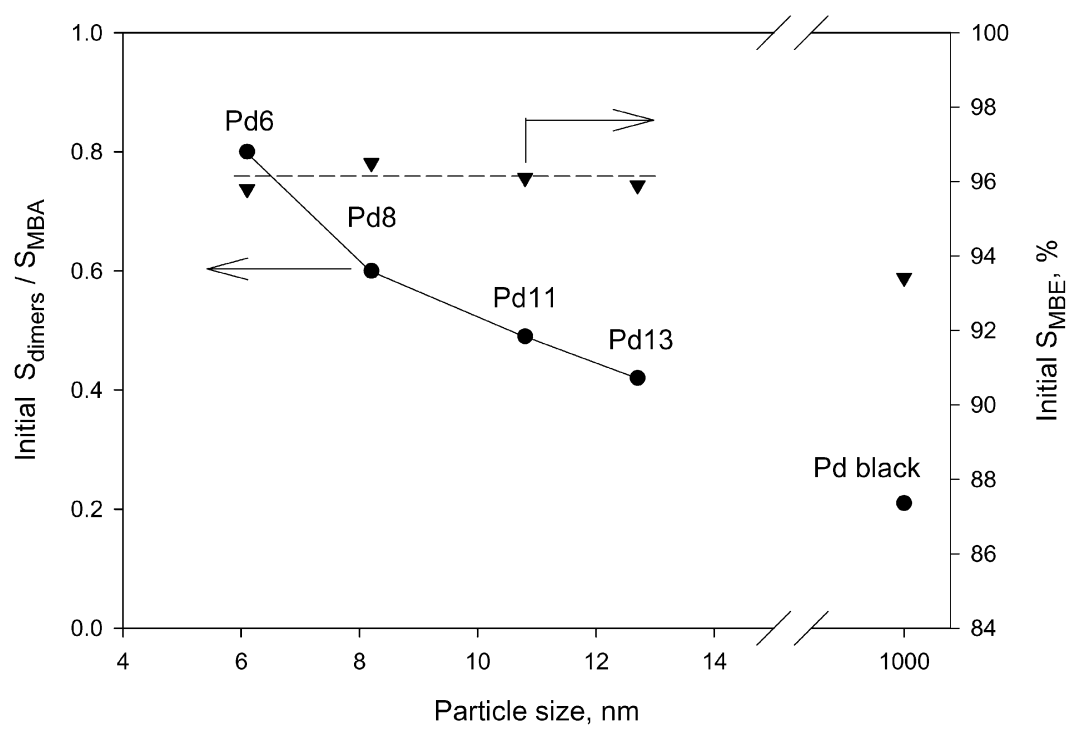

Fig. 8. Size dependence of initial $S_{\text {dimers }} / S_{\mathrm{MBA}}$ ratio $\left(\bullet\right.$, left axis) and $S_{\mathrm{MBE}}(\boldsymbol{\nabla}$, right axis). Reaction conditions: see Figs. 6, 7.

reaction. Further increase of Pd particle size almost does not influence TOF. Thus, for Pd13 catalyst with a particle diameter of $13 \mathrm{~nm}$, a TOF of $30 \mathrm{~s}^{-1}$ was determined. For comparison, palladium black with particle diameters of about $1 \mu \mathrm{m}$ exhibited a TOF of $35 \mathrm{~s}^{-1}$. However, as was shown for a number of Pd-catalyzed structure-sensitive reactions [2,8], the dependence of TOF on the particle size disappears when specific surface atoms only are taken into consideration. These surface atoms are then supposed to be the active sites. To determine the nature of the sites responsible for MBY hydrogenation, statistics of the surface atoms on fcc cubo-octahedron metal crystals were used [7]. The only type of surface atoms for which the ratio per surface atoms increases almost twice at the particle size increase from 6 to $13 \mathrm{~nm}$, are the atoms located on the facets of a (111) plane of fcc cubo-octahedron max- $\mathrm{B}_{5}$ crystals and surrounded by 9 nearest neighbors with 3 missing ones $\left(C_{9}^{3}\right.$ atoms [7]). Indeed, TOF calculation of MBY hydrogenation per number of $\mathrm{C}_{9}^{3}$ atoms showed independence on the particle size (Fig. 7). Similarly, Pd atoms located on (111) facets of fcc crystals were recently shown to be the active sites of 1,3-butadiene hydrogenation. This allowed the authors to claim the reaction as structure-sensitive but size-independent [51]. Apparent size dependence in 1,3-butadiene [51] and MBY hydrogenations is explained by a change in the nanoparticle surface structure with particle size variation.

Another point of interest in the MBY hydrogenation reaction is a study of the particle size effect on selectivity. Fig. 8 shows the independence of MBE selectivity on particle size in the range of 6-13 $\mathrm{nm}$. However, byproduct distribution varies with Pd size. The ratio of initial $S_{\text {dimers }} / S_{\mathrm{MBA}}$ decreases twice down to 0.4 with a particle size increase from 6 to $13 \mathrm{~nm}$. The use of larger particles (micrometer-sized Pd black) still does 
not allow elimination of the formation of dimers, giving an $S_{\text {dimers }} / S_{\text {MBA }}$ ratio of 0.2 . Again, a similar effect was found for 1,3-butadiene hydrogenation; the more atoms that were located on $\operatorname{Pd}(111)$ facets, the lower the ratio of isomerization to the saturated products [51]. In this work we showed for the first time that by varying the Pd particle size, it is possible to control the formation of different byproducts during hydrogenation of an acetylenic alcohol.

\section{Conclusion}

1. A simple method for preparing monodispersed metal nanoparticles was elaborated on the basis of the reverse $\mathrm{ME}$ technique. It allows recovery of the ME liquid phase and surfactant. The obtained nanoparticles suspended in a reaction substrate are suggested to be a useful tool in studying size effects in various liquid-phase reactions. The results are not influenced by the support effects and internal mass transport.

2. Monodispersed fcc Pd nanoparticles of 6, 8, 11, and $13 \mathrm{~nm}$ diameter were synthesized in reverse ME of water/ AOT/isooctane, purified from isooctane and AOT, and used as a probe for size effects in solvent-free hydrogenation of 2-methyl-3-butyn-2-ol.

3. The catalytic activity in MBY hydrogenation, expressed as initial TOF calculated per total surface atoms, increases with Pd particle size but remains constant if expressed in terms of number of specific atoms (active sites) on $\operatorname{Pd}(111)$ facets.

4. Particle size does not influence the selectivity to MBE but does affect the formation of dimers and saturated byproducts; the larger the particle size, the lower the ratio of dimers to MBA. This allows control of the formation of different byproducts during acetylene alcohol hydrogenation.

5. MBY hydrogenation is suggested to be a structure-sensitive but size-independent reaction for Pd particle sizes of $6-$ $13 \mathrm{~nm}$.

\section{Acknowledgments}

Financial support from the Swiss National Science Foundation (SNSF) and Commission for Technology and Innovation (CTI) is gratefully acknowledged. The authors thank Nicolas Xanthopoulos (EPFL-STI-IMX-LMCH) for the XPS measurements.

\section{References}

[1] R. Narayanan, M.A. El-Sayed, J. Phys. Chem. B 109 (2005) 12663.

[2] J. Le Bars, U. Specht, J.S. Bradley, D.G. Blackmond, Langmuir 15 (1999) 7621.

[3] T. Teranishi, M. Miyake, Chem. Mater. 10 (1998) 594.

[4] R.L. Augustine, S.T. O’Leary, J. Mol. Catal. A 95 (1995) 277.

[5] B. Coq, F. Figueras, J. Mol. Catal. A 173 (2001) 117.

[6] A. Molnar, A. Sarkany, M. Varga, J. Mol. Catal. A 173 (2001) 185.

[7] R. Van Hardeveld, F. Hartog, Surf. Sci. 15 (1969) 189.

[8] Y. Li, E. Boone, M.A. El-Sayed, Langmuir 18 (2002) 4921.
[9] C.O. Bennett, M. Che, J. Catal. 120 (1989) 293.

[10] M.M. Telkar, C.V. Rode, R.V. Chaudhari, S.S. Joshi, A.N. Nalawade, Appl. Catal. A 273 (2004) 11.

[11] V. Pillai, P. Kumar, M.J. Hou, P. Ayyub, D.O. Shah, Adv. Colloid Interface Sci. 55 (1995) 241.

[12] S. Eriksson, U. Nylen, S. Rojas, M. Boutonnet, Appl. Catal. A 265 (2004) 207.

[13] M. Boutonnet, J. Kizling, P. Stenius, G. Maire, Colloids Surf. 5 (1982) 209.

[14] D.G. Shchukin, G.B. Sukhorukov, Adv. Mater. 16 (2004) 671.

[15] M. Lade, H. Mays, J. Schmidt, R. Willumeit, R. Schomacker, Colloids Surf. A 163 (2000) 3.

[16] M. Yashima, L.K.L. Falk, A.E.C. Palmqvist, K. Holmberg, J. Colloid Interface Sci. 268 (2003) 348.

[17] M. Kishida, K. Umakoshi, J. Ishiyama, H. Nagata, K. Wakabayashi, Catal. Today 29 (1996) 355.

[18] D.M. de Jesus, M. Spiro, Langmuir 16 (2000) 4896.

[19] B. Yoon, H. Kim, C.M. Wai, Chem. Commun. (2003) 1040.

[20] M. Boutonnet, J. Kizling, R. Touroude, G. Maire, P. Stenius, Appl. Catal. 20 (1986) 163.

[21] H. Sato, T. Ohtsu, I. Komasawa, J. Chem. Eng. Jpn. 35 (2002) 255.

[22] H. Ohde, C.M. Wai, H. Kim, J. Kim, M. Ohde, J. Am. Chem. Soc. 124 (2002) 4540 .

[23] D. Astruc, F. Lu, J.R. Aranzaes, Angew. Chem. Int. Ed. 44 (2005) 7852

[24] Y. Berkovich, N. Garti, Colloids Surf. A 128 (1997) 91.

[25] J.H. Clint, I.R. Collins, J.A. Williams, B.H. Robinson, T.F. Towey, P.C.A. Khan-Lodhi, Faraday Discuss. 95 (1993) 219.

[26] N.M. Correa, D.H. Zorzan, M. Chiarini, G. Cerichelli, J. Org. Chem. 69 (2004) 8224.

[27] N.M. Correa, D.H. Zorzan, L. D’Anteo, E. Lasta, M. Chiarini, G. Cerichelli, J. Org. Chem. 69 (2004) 8231.

[28] V. Arcoleo, M. Goffredi, A. Longo, V. Turco Liveri, Mater. Sci. Eng. C 6 (1998) 7.

[29] A.J. Zarur, J.Y. Ying, Nature 403 (2000) 65.

[30] R.J. Higgins, R.L. Goldsmith, Patent WO 99/11243, 1999.

[31] J. Agrell, G. Germani, S.G. Jaras, M. Boutonnet, Appl. Catal. A 242 (2003) 233.

[32] M. Boutonnet, J. Kizling, V. Mintsa-Eya, A. Choplin, R. Toutode, G. Maire, P. Stenius, J. Catal. 103 (1987) 95.

[33] C. Wang, X. Guo, X. Wang, R. Wang, J. Hao, Catal. Lett. 96 (2004) 79.

[34] W.M. Targos, US Patent 4,714,693, 1987.

[35] R.S. Tanke, Patent WO 97/33690, 1997.

[36] M.B. Kizling, S. Jaras, L.J. Pettersson, U. Winter, M. Zwinkels, Patent WO 97/09114, 1997.

[37] T. Hanaoka, H. Hayashi, T. Tago, M. Kishida, K. Wakabayashi, J. Colloid Interface Sci. 235 (2001) 235.

[38] S. Xu, R. Zhao, X. Wang, Fuel Proc. Technol. 86 (2004) 123.

[39] O.P. Yadav, A. Palmqvist, N. Cruise, K. Holmberg, Colloids Surf. A 221 (2003) 131

[40] H. Abrevaya, W.M. Targos, US Patent 4,714,692, 1987.

[41] K. Zhang, C.H. Chew, S. Kawi, J. Wang, L.M. Gan, Catal. Lett. 64 (2000) 179.

[42] J.P. Boitiaux, J. Cosyns, S. Vasudevan, Appl. Catal. 6 (1983) 41.

[43] A. Benedetti, G. Fagherazzi, F. Pinna, G. Rampazzo, M. Selva, G. Strukul, Catal. Lett. 10 (1991) 215.

[44] A. Molnar, G.V. Smith, M. Bartok, J. Catal. 101 (1986) 67.

[45] E. Joannet, C. Horny, L. Kiwi-Minsker, A. Renken, Chem. Eng. Sci. 57 (2002) 3453 .

[46] E. Joannet, Ph.D. thesis, EPF, Lausanne, 2004.

[47] N.A. Zakarina, G.D. Zakumbaeva, N.F. Toktabaeva, B.B. Dyusenbina, E.N. Litvyakova, A.S. Kuanyshev, Kinet. Catal. 24 (1983) 733.

[48] G.D. Zakumbaeva, N.A. Zakarina, V.A. Naidin, A.M. Dostiyarov, N.F Toktabaeva, E.N. Litvyakova, Kinet. Catal. 24 (1983) 379.

[49] D.-H. Chen, C.-C. Wang, T.-C. Huang, J. Colloid Interface Sci. 210 (1999) 123.

[50] L. Kiwi-Minsker, E. Joannet, A. Renken, Chem. Eng. Sci. 59 (2004) 4919.

[51] J. Silvestre-Albero, G. Rupprechter, H.-J. Freund, Chem. Commun. (2006) 80. 